\title{
Recovery and stable persistence of chloroquine sensitivity in Plasmodium falciparum parasites after its discontinued use in Northern Uganda.
}

Betty Balikagala

Juntendo Daigaku

Miki Sakurai Yatsushiro

Tokyo Joshi lka Daigaku

Shin-Ichiro Tachibana

Juntendo Daigaku

Mie lkeda

Juntendo Daigaku

Masato Yamauchi

Juntendo Daigaku

Osbert T. Katuro

Mildmay Uganda

Edward Hosea Ntege

Ehime Daigaku Kogakubu Daigakuin Rikogaku Kenkyuka

Makoto Sekihara

Juntendo Daigaku

Naoyuki Fukuda

Juntendo Daigaku

Nobuyuki Takahashi

Tokyo Joshi lka Daigaku

Shouki Yatsushiro

Sangyo Gijutsu Sogo Kenkyujo Shikoku Center Kenko Kogaku Kenkyu Bumon

Toshiyuki Mori

Juntendo Daigaku

Makoto Hirai

Juntendo Daigaku

Walter Opio

Lacor Hospital

Paul S. Obwoya

Lacor Hospital

Denis Arony Anywar 
Gulu University Faculty of Science

\section{Mary Alai Auma}

Lacor Hospital

\section{Nirianne Marie Querijero Palacpac}

Research Institute for Microbial Disease Osaka University

\section{Takafumi Tsuboi}

Ehime Daigaku Kogakubu Daigakuin Rikogaku Kenkyuka

\section{Emmanuel Igwaro Odongo-Aginya}

Gulu University Faculty of Science

\section{Eisaku Kimura}

Nagasaki Daigaku

Martin David Ogwang

Lacor Hospital

\section{Toshihiro Horii}

Research Institute for Microbial Disease, Osaka University

Toshihiro Mita ( $\nabla$ tmita@juntendo.ac.jp)

Juntendo university https://orcid.org/0000-0001-8180-2344

\section{Research}

Keywords: Chloroquine sensitivity, Persistent recovery, Plasmodium falciparum, Uganda

Posted Date: November 28th, 2019

DOI: https://doi.org/10.21203/rs.2.17892/v1

License: (1) This work is licensed under a Creative Commons Attribution 4.0 International License.

Read Full License 


\section{Abstract}

Background Usage of chloroquine was discontinued from the treatment of Plasmodium falciparum infection in almost all endemic regions because of global spread of resistant parasites. Since the first report in Malawi, numerous epidemiological studies have demonstrated that the discontinuance led to reemergence of chloroquine-susceptible P. falciparum parasites, suggesting a possible role in future malaria control. However, most studies were cross-sectional, with few studies looking at the persistence of chloroquine recovery in long term. This study fills the gap by providing, for a period of at least 6 years, proof of persistent re-emergence/stable recovery of susceptible parasite populations using both molecular and phenotypic methods

Methods Ex vivo drug-susceptibility assays to chloroquine $(n=319)$ and lumefantrine $(n=335)$ were performed from 2013 to 2018 in Gulu, Northern Uganda where chloroquine had been removed from the official malaria treatment regimen since 2006. Genotyping of pfcrt and pfmdr1 was also performed.

Results Chloroquine resistance ( $\left.{ }^{3} 100 \mathrm{nM}\right)$ was observed in only $3(1.3 \%)$ samples. Average IC50 values for chloroquine were persistently low throughout the study period (17.4-24.9 nM). Parasites harboring pfcrt K76 alleles showed significantly lower IC50s to chloroquine than the parasites harboring K76T alleles (21.4 nM vs $43.1 \mathrm{nM}$, p-value $=3.9 \times 10-8)$. Prevalence of $\mathrm{K} 76$ alleles gradually increased from $71 \%$ in 2013 to $100 \%$ in 2018.

Conclusion This study found evidence of stable persistence of chloroquine susceptibility with the fixation of pfcrt K76 in Northern Uganda after discontinuation of chloroquine in the region. Accumulation of similar evidence in other endemic areas in Uganda could open channels for possible future re-use of chloroquine as an option for malaria treatment or prevention.

\section{Introduction}

Since the late 1940s, chloroquine was the mainstay for the treatment of Plasmodium falciparum infection. Heavy use of chloroquine, however, led to the emergence of $P$. falciparum parasites resistant to chloroquine in Southeast Asia and South America. The resistant parasites that first emerged in Southeast Asia spread to East Africa (Tanzania and Kenya) by $1980(1,2)$ and eventually across the malaria endemic regions of Africa (3). Chloroquine was therefore withdrawn/discontinued for routine treatment of $P$. falciparum in nearly all malaria endemic regions. However, with widespread discontinued use, numerous molecular-epidemiological studies showed that there was return of chloroquine susceptibility in $P$. falciparum field isolates (4). This is supported by ex vivo (5-16) and in vivo drug-susceptibility studies $(5,17,18)$. Findings suggest that chloroquine might be re-used in the future as an option for the treatment and/or chemoprophylaxis on the condition that chloroquine sensitivity is maintained in the area. As the parasite is "skilled" in evading antimalarial treatments, continuous surveillance on longitudinal persistence of chloroquine susceptibility by molecular and phenotypic analysis $(8,16,18,19)$ is needed. 
In Uganda, first-line treatment for uncomplicated malaria was changed from chloroquine to chloroquine plus sulfadoxine/pyrimethamine in 2000, then again changed to artemether lumefantrine in 2006 (20). Several studies in different regions in Uganda reported high prevalence of ex vivo chloroquine resistant parasites $\left(\mathrm{IC}_{50} \mathrm{~S} \geq 100 \mathrm{nM}\right)$ accompanied with high prevalence of lysine to threonine change at position 76 (K76T) in pfcrt (21-24). However, a recent study showed recovery of chloroquine susceptibility in Eastern Uganda, Tororo; average $\mathrm{IC}_{50}$ s decreased from $248 \mathrm{nM}$ in 2010-2013 to $33 \mathrm{nM}$ in the community and $57 \mathrm{nM}$ in the hospital setting in 2016 (10). Here, to investigate whether chloroquine sensitivity also recovered in other regions in Uganda and, if so, to examine the persistence of chloroquine sensitivity, ex vivo drug susceptibility studies for a six-year period since 2013 in Gulu, Northern Uganda were performed. Our results show that chloroquine susceptibility stably persisted during the study period with a significant decrease and eventual absence of the chloroquine resistant K76T alleles in pfcrt.

\section{Materials And Methods}

\section{Study site}

A comprehensive drug susceptibility assessment was conducted at St. Mary's Hospital Lacor in Gulu, Northern Uganda (Fig 1) from 2013 to 2018 (25, 26): Oct-Nov 2013, May-Jun and Oct-Nov 2014, May-Jun and Oct 2015, Jun-Jul and Oct-Nov 2016, Jun 2017 and Jun 2018. Average temperature in the studied area is $24.6^{\circ} \mathrm{C}$ and average annual rainfall is about $1507 \mathrm{~mm}$ with two rainy seasons; a smaller peak in April-May (average rainfall $150 \mathrm{~mm}$ ) and a heavier peak in August-September (average rainfall $234 \mathrm{~mm}$ ) (27). P. falciparum is the most prevalent species and is mainly transmitted by Anopheles fenestus as the major vector and An. gambiae.

Malaria control programs in the studied region include vector control by long-lasting insecticidal nets (LLINs) and indoor residual spraying (IRS), artemisinin combination therapy (ACT) together with improved diagnosis, management of severe malaria, and intermittent preventive treatment of malaria during pregnancy. These control measures were performed with funding from the Global Fund, USAID/PMI, DFID, World Vision and other partners (28). Mass distribution of LLINs was first implemented in 2009-2010, which continued until 2013-2014. Through these extensive efforts, malaria burden was effectively reduced from $72 \%$ in 2009 to $29 \%$ in 2014 (27).

\section{Patients}

Initial screening was performed by using RDT (SD BIOLINE Malaria Ag P.f/Pan test, Abbott, USA) for 1575 symptomatic patients who visited St. Mary's Hospital Lacor. Inclusion criteria were: (a) patients that are $P$. falciparum positive by RDT, (b) aged $\geq 6$ months, and (c) with no history of taking antimalarial drug(s) within two weeks before enrollment. Patients fulfilling the inclusion criteria were enrolled after obtaining written informed consent from the patients or parent/guardian(s). For children aged 7 to 17 years, separate assent was also obtained. 


\section{Sample collection and ex vivo susceptibility assay for chloroquine and lumefantrine}

Blood samples of approximately $100-500 \mu \mathrm{L}$ (<2 years old), and $1 \mathrm{ml}$ ( $\geq 2$ years old) were collected by peripheral venipuncture or finger prick and immediately transferred to the laboratory adjacent to the hospital. Thick and thin blood smears stained for 30 min with $2 \%$ Giemsa solution were used to determine parasitemia.

At every visit from 2013-2018 (a total of nine times sampling period), ex vivo drug susceptibility studies were performed. Ex vivo susceptibility was evaluated for chloroquine and lumefantrine for the samples with parasitemia $\geq 0.05 \%$ as previously reported (29). Parasite culture was incubated in the presence of chloroquine $(25-1600 \mathrm{nM})$ or lumefantrine $(1.25-80 \mathrm{nM})$ at $37^{\circ} \mathrm{C}$ for $72 \mathrm{~h}$ in a gas atmosphere of $5 \%$ $\mathrm{CO}_{2}, 5 \% \mathrm{O}_{2}$ (AnaeroPack malaria culture system, Mitsubishi Gas Chemical Co. Inc., Tokyo, Japan). Laboratory-maintained 3D7 clone was used for quality evaluation of pre-dosed drug plates. Parasite culture without antimalarials served as control. To evaluate parasite growth, thick smears were made from drug free culture after $72 \mathrm{~h}$ of incubation and the number of schizonts were counted. If less than 5 schizonts were seen per field, the test samples included in that plate were not used for further analysis. Drug sensitivity was assessed using an enzyme-linked immunosorbent assay (ELISA) that quantifies parasite histidine-rich protein-2 (HRP-2) (30). The effective concentration needed to inhibit $P$. falciparum growth by $50 \%\left(\mathrm{IC}_{50}\right)$ was determined by non-linear regression using an online ICEstimator software (www.antimalarial-icestimator.net) (31). The quality of the ex vivo drug assay was evaluated based upon the level of fitness to the expected shape of the curve obtained by the inhibitory sigmoid Emax model (31).

\section{Pfcrt and pfmdr1 genotyping}

Polymorphisms at amino acid positions 72-76 in pfcrt were determined by direct sequencing. In $P$. falciparum multidrug resistance-1 (pfmdr1), polymorphisms at codons 86, 184, 1034, 1042 and 1246 were determined by direct sequencing and/or restriction fragment length polymorphism (RFLP) analysis, as previously described $(29,32)$. For direct sequencing, initial and nested PCR was done with PrimeSTAR Max DNA Polymerase (Takara Bio Inc., Japan) in $10 \mu \mathrm{L}$ reaction mixture containing $1 \mu \mathrm{L}$ of DNA template and $0.5 \mu \mathrm{M}$ of each primer set. Excess primers and unincorporated nucleotides from the nested PCR product were enzymatically removed with ExoSAP-IT Kit (Amersham Biosciences, Buckinghamshire, UK) and direct sequence was performed $\left(96^{\circ} \mathrm{C}\right.$ for $1 \mathrm{~min}, 25$ cycles of $96^{\circ} \mathrm{C}$ for $30 \mathrm{~s}, 50^{\circ} \mathrm{C}$ for 30 sec and $60^{\circ} \mathrm{C}$ for $4 \mathrm{~min}$, and a final cycle at $60^{\circ} \mathrm{C}$ for $1 \mathrm{~min}$ ) with a BigDye Terminator v1.1 cycle sequencing kit in the 
Applied Biosystems 3130/3130xL genetic analyzer (Life Technologies, Carlsbad, California, U.S.A). Samples with overlapping peaks of at least $50 \%$ in height were considered harboring mixed genotypes.

\section{Full sequencing of pfcrt}

The entire sequence of pfcrt was obtained using target sequencing $(n=39)$ and whole-genome sequence $(n=17)$ as previously reported (26). For target sequencing, the DNA fragment of a genomic region coding for $p f c r t$ gene was amplified by PCR with primers (Pfcrt-F: 5'-TAC TTT CCC AAG TTG TAC TGC TTC TAA GCT-3', Pfcrt-R: 5'-TTT ACC TAT TTA TCA AAA CAC CAA AAG GGA-3'), which covers the whole DNA sequence of pfcrt gene. PCR was performed with PrimeSTAR GXL DNA Polymerase (Takara Bio Inc., Japan) in $5 \mu \mathrm{L}$ reaction mixture containing $1 \mu \mathrm{L}$ of DNA solution and $0.25 \mu \mathrm{M}$ of primer set. PCR conditions consisted of denaturation at $98^{\circ} \mathrm{C}$ for $10 \mathrm{~s}$, followed by 40 cycles of amplification $\left(98^{\circ} \mathrm{C}\right.$ for 10 $\mathrm{s}, 60^{\circ} \mathrm{C}$ for $15 \mathrm{~s}$, and $68^{\circ} \mathrm{C}$ for $5 \mathrm{~min}$ ), with a final elongation period of $68^{\circ} \mathrm{C}$ for $5 \mathrm{~min}$. PCR products were diluted with $5 \mu \mathrm{L}$ of pure water, electrophoresed in $2 \%$ agarose gel and stained with ethidium bromide. The PCR products were then purified with the ExoSAP-IT reagent (Affymetrix, USA). Libraries were prepared from the purified PCR products with Nextera XT DNA Library Prep Kit (Illumina, USA). The libraries were sequenced by MiSeq (Illumina) with the paired-end method and $250 \mathrm{bp}$ of read length. The reads were also used to map the pfcrt gene sequence of $P$. falciparum 3D7 as a reference and assembled a single contiguous sequence by CLC Genomics Workbench (Qiagen). All sequences were deposited in the DNA Data Bank of Japan (DDBJ) with accession numbers LC498195-LC498250.

\section{Statistical analysis}

All statistical analyses were performed using R software (version 3.6.1). Data was analyzed using Kruskall Wallis test, Wilcoxon rank sum test, and Jonckheere-Terpstra test. $P$ values $<0.05$ were considered statistically significant.

\section{Results}

\section{Ex vivo drug susceptibility of chloroquine and lumefantrine}

Of 1575 patients who visited St. Mary's Hospital Lacor, 793 patients were enrolled based on $P$. falciparumpositive results by RDT (Fig 2). The rest were excluded because of (a) absence of $P$. falciparum by microscopic examination ( $n=535)$, (b) use of antimalarial drug(s) within the last two weeks before enrollment $(n=198)$, or (c) other reasons $(n=49)$ (Fig. 2). The commonly used antimalarial for pretreatment was artemether-lumefantrine (77\%) (Table 3 ). Chloroquine use was confirmed in only 3 patients in 2013 and one patient in 2014. 
Among 793 blood samples obtained from enrolled patients, 203 were excluded because of very low parasitemia $(<0.05 \%)$ or insufficient amount of blood, resulting in 590 samples used for ex vivo drug susceptibility assays. Also, ex vivo study for chloroquine in 2016 and lumefantrine in 2015 was not performed because of inadequate quality of pre-dosed drug plates. Thus, in total, 319 and 335 ex vivo drug-susceptibility assays for chloroquine and lumefantrine, respectively were available for analyses. Background information of patients who participated in the study per year is shown in Table 1. Median age was 3.5 years (IQR 2.0-4.8) and hemoglobin level $<10 \mathrm{~g} / \mathrm{dL}$ was observed in $27 \%$ of patients. Median parasitemia at enrollment were $0.2-3.5 \%$, which significantly varied between studied year ( $\mathrm{p}$ value $=3.910^{-15}$, Kruskal-Wallis test). Beside parasitemia at enrollment, no significant difference was observed in background factors among the studied years.

Of 319 and 335 ex vivo drug-susceptibility assay for chloroquine and lumefantrine, respectively, 42 chloroquine and 48 lumefantrine assays were performed with 0 instead of $A B$ blood-group serum due to the latter's unavailability during the sampling period. These samples were excluded from further analysis. Thus, in summary, ex vivo drug study was successfully conducted for $239 / 277$ samples (86.3\%) for chloroquine and $168 / 287$ for lumefantrine (58.5\%) (Fig 2). For chloroquine, only $1.3 \%(3 / 239)$ fulfilled the criteria for chloroquine resistance $\left(\mathrm{IC}_{50}>100 \mathrm{nM}\right)$ (Fig 3a). From 2013-2018, the geometric means of the $\mathrm{IC}_{50} \mathrm{~s}(17.4-24.9 \mathrm{nM})$ were much lower than the threshold for chloroquine resistance and was stable without any significant decrease or increase in trend throughout the study period ( $p$-value $=0.32$ Jonckheere-Terpstra test). The highest $\mathrm{IC}_{50}$ was $148.8 \mathrm{nM}$, observed in 2015 in a 9-year-old girl. For lumefantrine, $\mathrm{IC}_{50}$ s displayed no specific trend over time ranging from $20.5 \mathrm{nM}$ to $32.0 \mathrm{nM}$ (p-value = 0.16 , Jonckheere-Terpstra test). In all studied parasites, $I_{50}$ values were below the conservative cut-off of $50 \mathrm{nM}$ for lumefantrine resistance (33), and lower than the $150 \mathrm{nM}$ value (34)(Fig 3b)

\section{Allele prevalence and frequency of pfcrt and pfmdr1 polymorphisms}

In pfcrt, a prevalence of chloroquine-resistant alleles (CVIET allele; amino acid position 72-76, mutation underlined) significantly decreased from $28.8 \%$ in 2013 to $1.1 \%$ in 2016 and finally cannot be detected in 2017 (Fig 4). In pfmdr1, chloroquine sensitive N86 allele was fixed or nearly fixed throughout the study period. Prevalence of the mutant allele at position 184 (Y184F) gradually increased from $2.4 \%$ in 2013 to $48.5 \%$ in 2018 , albeit this trend was not significant ( $p$-value $=0.13$, Jonckheere-Terpstra trend test). Wildtype alleles were nearly fixed at other loci in pfmdr1.

\section{Association between ex vivo drug sensitivity and alleles in pfcrt and pfmdr1}


In pfcrt, parasites carrying wild-type alleles showed significantly lower $\mathrm{IC}_{50}$ s to chloroquine than those carrying mutant allele (geometric mean, 21.4 vs $43.1 \mathrm{nM}$, p-value $3.910^{-8}$, Wilcoxon rank sum test) (Fig 5). By sequencing the entire pfcrt in 44 randomly selected samples to see whether other polymorphism(s) besides those at position 72-76 played a role in the recovery of chloroquine susceptibility (Table 2), 31 (71\%) samples bear only wild-type alleles of the gene. The second $(n=5,11 \%)$ most common haplotype, $\mathrm{HP}-4$, corresponds to a prevalent mutant haplotype (CVMNT+A220S+Q271E+R371I) in Africa $(3,35)$.

Remaining eight parasites harbored minor haplotypes, all with wild type allele $\mathrm{K} 76$ and displayed $\mathrm{IC}_{50 \text { s }}$ to chloroquine of 18-35 $\mathrm{nM}$. A previous study (16), has implicated C356R in the sensitivity recovery of chloroquine in the K76T-harboring parasites, however this mutant allele was not found in the study area. These results further confirm the expansion of wild K76-harboring parasites as the cause of chloroquine susceptibility reversal in our study area, rather than additional mutation in the pfcrt gene.

Association between ex vivo drug sensitivity and respective $p f m d r 1$ alleles, however, could not be properly evaluated because of substantial deviation in allele frequencies except for position 184. At this position, no significant difference in chloroquine $\mathrm{IC}_{50 \mathrm{~s}}$ was observed between wild and mutant alleles $(25.3 \mathrm{nM}$ vs $22.0 \mathrm{nM}, \mathrm{p}$-value $=0.192$, Wilcoxon rank sum test) (Fig 5). Analysis of susceptibility to lumefantrine indicated that parasites carrying pfcrt CVIET exhibited significantly lower IC $\mathrm{I}_{50} \mathrm{~S}$ than those with CVMNK (13.4 vs $28.0 \mathrm{nM}$, p-value $=8.110^{-5}$, Wilcoxon rank sum test) (Fig 5). No significant difference in $\mathrm{IC}_{50} \mathrm{~s}$ for lumefantrine was found between parasites with pfmdr1 Y184 (25.0 nM) and Y184F (30.0 nM).

\section{Discussion}

In Uganda, chloroquine was officially withdrawn and replaced with artemether-lumefantrine in 2006. The present analysis revealed that chloroquine susceptibility has returned and is stably maintained for at least six-years in Gulu, Northern Uganda. To the best of our knowledge, this is the first report in Uganda to demonstrate stable and persistent recovery of chloroquine sensitivity using both phenotypic and genotypic approaches.

The prevalence of K76 allele in pfcrt rapidly increased from 67\% in 2013 to complete fixation in 2017. This is most probably due to K76-harboring parasites outcompeting the K76T-harboring parasites because of fitness advantage in the absence of chloroquine selection pressure, as previously observed in Malawi (36) (37). Indeed, recent transfection studies also show that K76T confers a substantial fitness cost to parasites $(35,38)$. This fitness cost can be partly explained by a functional impairment in haemoglobin digestion and subsequent reduction in the supply of amino acids in K76T harbouring parasites (39). In another possible scenario, a back mutation from $\mathrm{T}$ to $\mathrm{K}$ at position 76 in pfcrt may potentially induce chloroquine sensitivity. As an example, the chloroquine-susceptible 106/1 clone had mutant alleles at positions 74 and 75 but showed wild-type K allele at position 76 (CVIEK) (40). The only difference in the pfcrt haplotype at position 72-76 in this clone and the widely prevalent chloroquineresistant haplotype (CVIET) is at amino acid position 76. In the present analysis, however, no such haplotype (CVIEK) was found, negating this possibility. Also, no evidence of additional mutation in pfcrt 
such as C350R which has been reported to be associated with the restoration of CQ susceptibility (16) was obtained. Taken together, these results strongly suggest that neither back mutation nor additional mutations in pfcrt was associated with the observed recovery of chloroquine sensitivity in the study area.

It is of note that recovery of chloroquine sensitivity after its withdrawal occurred much earlier in Gulu than in other regions in Uganda $(9,22-24,41)$. In 2013 , as much as $65 \%$ of parasites displayed ex vivo chloroquine resistance (24) and 60-80\% carried K76T allele in Tororo, Eastern Uganda (42). In contrast, present results revealed that prevalence of ex vivo chloroquine-resistance and K76T alleles were already $6 \%$ and $29 \%$, respectively in 2013 , indicating a faster recovery or re-emergence of chloroquine sensitive strains in the region. Despite the government's move to change the national treatment policy, chloroquine might be used as self-treatment and/or prophylaxis. Such chloroquine use potentially creates various levels of chloroquine selecting pressure in the region, which would be one of the important factors that affects the speed of recovery of susceptible parasites (43). However, countrywideantimalarial survey reported no considerable difference of chloroquine use between Tororo and our study area (44-46), suggesting that this could not be a main factor for the observed findings.

Piperaquine use in Tororo might explain the observed difference to some extent. Dihydroartemisininpiperaquine has been used as a second line treatment for uncomplicated malaria in Uganda. In Tororo, this regimen was widely used in different drug trials for malaria treatment $(47,48)$ and chemoprevention $(24,49-52)$. Previous studies indicated that dihydroartemisinin-piperaquine treatment selected the N86Y allele in pfmdr1 in Uganda $(48,52-54)$, albeit one study questioned this association (55). Approximately one third of parasites possessed the N86Y in 2010-2013 in Tororo (9), which was much higher than that found in our study area (2\%). Since N86Y is associated with chloroquine resistance, it might be plausible that the N86Y mutation selected by piperaquine played a role in the slower recovery of chloroquine sensitivity in Tororo.

In contrast to chloroquine, average lumefantrine $\mathrm{IC}_{50}$ values in the present analysis $(21-29 \mathrm{nM})$ were considerably higher than those in Eastern Uganda $(3.0-5.4 \mathrm{nM})(9,24)$. It has been suggested that decrease in lumefantrine susceptibility are associated with wild type alleles in pfcrt (K76) and pfmdr1 (N86 and D1246) (48, 56-59). In this study the higher $\mathrm{IC}_{50}$ s to lumefantrine were observed in $\mathrm{K} 76$ sequences than those with K76T. The higher prevalence of K76 allele in our study area than that in Eastern Uganda $(9,23,42)$ may partly explain the observed lumefantrine susceptibility.

\section{Conclusions}

The study shows the stable persistence of chloroquine-susceptibility with the fixation of pfcrt K76 in Northern Uganda. This observation implies the possibility of future clinical trials for potential re-use of chloroquine as an option for malaria treatment or prevention. Such trial was performed in Malawi where longtime stable chloroquine susceptibility has been evidenced and has revealed that weekly chemoprophylaxis with chloroquine showed $78 \%$ lower risk of clinical malaria than intermittent sulfadoxine-pyrimethamine (60). Similar trial in Uganda would provide an insight into potential re- 
introduction of chloroquine. However, further evidence of long-time persistence of return of chloroquine susceptibility is warranted in other endemic areas in Uganda before the implementation of clinical trials.

\section{Declarations}

\section{Acknowledgment}

We specially thank study participants, participants' guardians for consent to participate in the study as well as study teams from St. Mary's Hospital Lacor and the Department of Tropical Medicine and Parasitology Juntendo University for sample collection. We also thank Hiroaki Nakanishi and Kazuyuki Saito for technical assistance.

\section{Authors' contributions}

$\mathrm{BB}, \mathrm{TH}$ and TMita designed and coordinated the study; BB, MSY, SIT, MI, MY, OTK, EHN, MS, NF, SY, WO, PSO, DAA, MAA, NMQP, TT, EIO, EK, MO, TH and TMita performed the field study; BB, MSY, SIT, MI, MY, NF, $\mathrm{NT}, \mathrm{MH}$ and TMori performed the laboratory work; BB and TMita analyzed and interpreted the data; $\mathrm{BB}$, NMQP and TMita wrote the manuscript. All authors contributed significantly to this work. All authors read and approved the final manuscript.

\section{Competing interests}

All authors declare no competing interests.

\section{Availability of data and materials}

The primary datasets used and analyzed during the current study are available from the corresponding author on reasonable request.

\section{Ethics approval and consent to participate}

Ethical approval for the study was obtained from Lacor Hospital Institutional Research and Ethics Committee (Ref; LHIREC 021/09/13), the Uganda National Council for Science and Technology (Ref; HS 1395), and Juntendo Research and Ethics Committee (Ref; 14-169)

\section{Consent for publication}


Prior to participation, all study subjects consented to the publication of study results in medical literature in an anonymized manner.

\section{Funding}

This study was financially supported by Grants-in-aid for scientific research [26460515, 26305015, 17H04074], Health and Labour Sciences Research Grants [H26-Iryokiki-Ippan-004] from Ministry of Health, Labour and Welfare (MEXT) of Japan, AMED under Grant Number JP15km0908001 and by the Global Health Innovative Technology (GHIT) Fund (G2015-210) awarded to Professor Toshihiro Mita. Further funding was from Strategic Promotion of International Cooperation to Accelerate Innovation in Africa (MEXT) (16jm0410004j0005) and the GHIT Fund (G2013-105) awarded to Prof Toshihiro Horii as well as from Juntendo University Collaborative Project Research Grant (PRO 2019-21) awarded to Dr. Betty Balikagala.

\section{References}

1.Campbell CC, Chin W, Collins WE, Teutsch SM, Moss DM. Chloroquine-resistant Plasmodium falciparum from East Africa: cultivation and drug sensitivity of the Tanzanian I/CDC strain from an American tourist. Lancet. 1979;2(8153):1151-4.

2.Fogh S, Jepsen S, Effersoe P. Chloroquine-resistant Plasmodium falciparum malaria in Kenya. Trans $\mathrm{R}$ Soc Trop Med Hyg. 1979;73(2):228-9.

3.Mita T, Tanabe K, Kita K. Spread and evolution of Plasmodium falciparum drug resistance. Parasitol Int. 2009;58(3):201-9.

4.Ocan M, Akena D, Nsobya S, Kamya MR, Senono R, Kinengyere AA, et al. Persistence of chloroquine resistance alleles in malaria endemic countries: a systematic review of burden and risk factors. Malar J. 2019;18(1):76.

5.Mita T, Kaneko A, Lum JK, Bwijo B, Takechi M, Zungu IL, et al. Recovery of chloroquine sensitivity and low prevalence of the Plasmodium falciparum chloroquine resistance transporter gene mutation K76T following the discontinuance of chloroquine use in Malawi. Am J Trop Med Hyg. 2003;68(4):413-5.

6.Akala HM, Eyase FL, Cheruiyot AC, Omondi AA, Ogutu BR, Waters NC, et al. Antimalarial drug sensitivity profile of western Kenya Plasmodium falciparum field isolates determined by a SYBR Green I in vitro assay and molecular analysis. Am J Trop Med Hyg. 2011;85(1):34-41.

7.Eyase FL, Akala HM, Ingasia L, Cheruiyot A, Omondi A, Okudo C, et al. The role of Pfmdr1 and Pfcrt in changing chloroquine, amodiaquine, mefloquine and lumefantrine susceptibility in western-Kenya $P$. falciparum samples during 2008-2011. PLoS One. 2013;8(5):e64299. 
8.Lucchi NW, Komino F, Okoth SA, Goldman I, Onyona P, Wiegand RE, et al. In Vitro and Molecular Surveillance for Antimalarial Drug Resistance in Plasmodium falciparum Parasites in Western Kenya Reveals Sustained Artemisinin Sensitivity and Increased Chloroquine Sensitivity. Antimicrob Agents Chemother. 2015;59(12):7540-7.

9.Rasmussen SA, Ceja FG, Conrad MD, Tumwebaze PK, Byaruhanga O, Katairo T, et al. Changing Antimalarial Drug Sensitivities in Uganda. Antimicrob Agents Chemother. 2017;61(12).

10.Fall B, Pascual A, Sarr FD, Wurtz N, Richard V, Baret E, et al. Plasmodium falciparum susceptibility to anti-malarial drugs in Dakar, Senegal, in 2010: an ex vivo and drug resistance molecular markers study. Malar J. 2013;12:107.

11.Issaka M, Salissou A, Arzika I, Guillebaud J, Maazou A, Specht S, et al. Ex vivo responses of Plasmodium falciparum clinical isolates to conventional and new antimalarial drugs in Niger. Antimicrob Agents Chemother. 2013;57(7):3415-9.

12.Quashie NB, Duah NO, Abuaku B, Quaye L, Ayanful-Torgby R, Akwoviah GA, et al. A SYBR Green 1based in vitro test of susceptibility of Ghanaian Plasmodium falciparum clinical isolates to a panel of anti-malarial drugs. Malar J. 2013;12:450.

13.Salissou A, Zamanka H, Biyghe Binze B, Rivière T, Tichit M, Ibrahim ML, et al. Low Prevalence of Pfcrt Resistance Alleles among Patients with Uncomplicated Falciparum Malaria in Niger Six Years after Chloroquine Withdrawal. Malar Res Treat. 2014;2014:614190.

14.Tinto $\mathrm{H}$, Bonkian LN, Nana LA, Yerbanga I, Lingani M, Kazienga A, et al. Ex vivo anti-malarial drugs sensitivity profile of Plasmodium falciparum field isolates from Burkina Faso five years after the national policy change. Malar J. 2014;13:207.

15.Mbaye A, Gaye A, Dieye B, Ndiaye YD, Bei AK, Affara M, et al. Ex vivo susceptibility and genotyping of Plasmodium falciparum isolates from Pikine, Senegal. Malar J. 2017;16(1):250.

16.Pelleau S, Moss EL, Dhingra SK, Volney B, Casteras J, Gabryszewski SJ, et al. Adaptive evolution of malaria parasites in French Guiana: Reversal of chloroquine resistance by acquisition of a mutation in pfcrt. Proc Natl Acad Sci U S A. 2015;112(37):11672-7.

17.Laufer MK, Thesing PC, Eddington ND, Masonga R, Dzinjalamala FK, Takala SL, et al. Return of chloroquine antimalarial efficacy in Malawi. N Engl J Med. 2006;355(19):1959-66.

18.Laufer MK, Thesing PC, Dzinjalamala FK, Nyirenda OM, Masonga R, Laurens MB, et al. A longitudinal trial comparing chloroquine as monotherapy or in combination with artesunate, azithromycin or atovaquone-proguanil to treat malaria. PLoS One. 2012;7(8):e42284.

19.Frosch AE, Laufer MK, Mathanga DP, Takala-Harrison S, Skarbinski J, Claassen CW, et al. Return of widespread chloroquine-sensitive Plasmodium falciparum to Malawi. J Infect Dis. 2014;210(7):1110-4. 
20.Nanyunja M, Nabyonga Orem J, Kato F, Kaggwa M, Katureebe C, Saweka J. Malaria treatment policy change and implementation: the case of Uganda. Malar Res Treat. 2011;2011:683167.

21.Nsobya SL, Kiggundu M, Nanyunja S, Joloba M, Greenhouse B, Rosenthal PJ. In vitro sensitivities of Plasmodium falciparum to different antimalarial drugs in Uganda. Antimicrob Agents Chemother. 2010;54(3):1200-6.

22.Kamugisha E, Bujila I, Lahdo M, Pello-Esso S, Minde M, Kongola G, et al. Large differences in prevalence of Pfcrt and Pfmdr1 mutations between Mwanza, Tanzania and Iganga, Uganda-a reflection of differences in policies regarding withdrawal of chloroquine? Acta Trop. 2012;121(2):148-51.

23.Mbogo GW, Nankoberanyi S, Tukwasibwe S, Baliraine FN, Nsobya SL, Conrad MD, et al. Temporal changes in prevalence of molecular markers mediating antimalarial drug resistance in a high malaria transmission setting in Uganda. Am J Trop Med Hyg. 2014;91(1):54-61.

24.Tumwebaze P, Conrad MD, Walakira A, LeClair N, Byaruhanga O, Nakazibwe C, et al. Impact of antimalarial treatment and chemoprevention on the drug sensitivity of malaria parasites isolated from ugandan children. Antimicrob Agents Chemother. 2015;59(6):3018-30.

25.Balikagala B, Mita T, Ikeda M, Sakurai M, Yatsushiro S, Takahashi N, et al. Absence of in vivo selection for K13 mutations after artemether-lumefantrine treatment in Uganda. Malar J. 2017;16(1):23.

26.Ikeda M, Kaneko M, Tachibana SI, Balikagala B, Sakurai-Yatsushiro M, Yatsushiro S, et al. ArtemisininResistant Plasmodium falciparum with High Survival Rates, Uganda, 2014-2016. Emerg Infect Dis. 2018;24(4):718-26.

27.Simple O, Mindra A, Obai G, Ovuga E, Odongo-Aginya El. Influence of Climatic Factors on Malaria Epidemic in Gulu District, Northern Uganda: A 10-Year Retrospective Study. Malar Res Treat. 2018;2018:5482136.

28.UBOS. Uganda Malaria Indicator Survey 2014-15. 2015

29.Sekihara M, Tachibana SI, Yamauchi M, Yatsushiro S, Tiwara S, Fukuda N, et al. Lack of significant recovery of chloroquine sensitivity in Plasmodium falciparum parasites following discontinuance of chloroquine use in Papua New Guinea. Malar J. 2018;17(1):434.

30.Noedl H, Wernsdorfer WH, Miller RS, Wongsrichanalai C. Histidine-rich protein II: a novel approach to malaria drug sensitivity testing. Antimicrob Agents Chemother. 2002;46(6):1658-64.

31.Le Nagard H, Vincent C, Mentré F, Le Bras J. Online analysis of in vitro resistance to antimalarial drugs through nonlinear regression. Comput Methods Programs Biomed. 2011;104(1):10-8.

32.Duraisingh MT, Jones P, Sambou I, von Seidlein L, Pinder M, Warhurst DC. The tyrosine-86 allele of the pfmdr1 gene of Plasmodium falciparum is associated with increased sensitivity to the anti-malarials 
mefloquine and artemisinin. Mol Biochem Parasitol. 2000;108(1):13-23.

33.Kaddouri H, Djimdé A, Dama S, Kodio A, Tekete M, Hubert V, et al. Baseline in vitro efficacy of ACT component drugs on Plasmodium falciparum clinical isolates from Mali. Int J Parasitol. 2008;38(7):7918.

34.Basco LK, Bickii J, Ringwald P. In vitro activity of lumefantrine (benflumetol) against clinical isolates of Plasmodium falciparum in Yaoundé, Cameroon. Antimicrob Agents Chemother. 1998;42(9):2347-51.

35.Dhingra SK, Gabryszewski SJ, Small-Saunders JL, Yeo T, Henrich PP, Mok S, et al. Global Spread of Mutant PfCRT and Its Pleiotropic Impact on Plasmodium falciparum Multidrug Resistance and Fitness. MBio. 2019;10(2).

36.Mita T, Kaneko A, Lum JK, Zungu IL, Tsukahara T, Eto H, et al. Expansion of wild type allele rather than back mutation in pfcrt explains the recent recovery of chloroquine sensitivity of Plasmodium falciparum in Malawi. Mol Biochem Parasitol. 2004;135(1):159-63.

37.Laufer MK, Takala-Harrison S, Dzinjalamala FK, Stine OC, Taylor TE, Plowe CV. Return of chloroquinesusceptible falciparum malaria in Malawi was a reexpansion of diverse susceptible parasites. J Infect Dis. 2010;202(5):801-8.

38.Petersen I, Gabryszewski SJ, Johnston GL, Dhingra SK, Ecker A, Lewis RE, et al. Balancing drug resistance and growth rates via compensatory mutations in the Plasmodium falciparum chloroquine resistance transporter. Mol Microbiol. 2015;97(2):381-95.

39.Lewis IA, Wacker M, Olszewski KL, Cobbold SA, Baska KS, Tan A, et al. Metabolic QTL analysis links chloroquine resistance in Plasmodium falciparum to impaired hemoglobin catabolism. PLoS Genet. 2014;10(1):e1004085.

40.Fidock DA, Nomura T, Talley AK, Cooper RA, Dzekunov SM, Ferdig MT, et al. Mutations in the P. falciparum digestive vacuole transmembrane protein PfCRT and evidence for their role in chloroquine resistance. Mol Cell. 2000;6(4):861-71.

41.Asua V, Vinden J, Conrad MD, Legac J, Kigozi SP, Kamya MR, et al. Changing Molecular Markers of Antimalarial Drug Sensitivity across Uganda. Antimicrob Agents Chemother. 2019;63(3).

42.Tumwebaze P, Tukwasibwe S, Taylor A, Conrad M, Ruhamyankaka E, Asua V, et al. Changing Antimalarial Drug Resistance Patterns Identified by Surveillance at Three Sites in Uganda. J Infect Dis. 2017;215(4):631-5.

43. Rosenthal PJ. The interplay between drug resistance and fitness in malaria parasites. Mol Microbiol. 2013;89(6):1025-38. 
44.Sears D, Kigozi R, Mpimbaza A, Kakeeto S, Sserwanga A, Staedke SG, et al. Anti-malarial prescription practices among outpatients with laboratory-confirmed malaria in the setting of a health facility-based sentinel site surveillance system in Uganda. Malar J. 2013;12:252.

45.ACTwatchGroup, Pace. ACTwatch study Reference Document: Uganda Outlet Survey 2013. Washington DC: PSI; 2014.

46.Sserwanga A, Sears D, Kapella BK, Kigozi R, Rubahika D, Staedke SG, et al. Anti-malarial prescription practices among children admitted to six public hospitals in Uganda from 2011 to 2013. Malar J. 2015;14:331.

47.4ABC_Group. A head-to-head comparison of four artemisinin-based combinations for treating uncomplicated malaria in African children: a randomized trial. PLoS Med; 2011.

48.Conrad MD, LeClair N, Arinaitwe E, Wanzira H, Kakuru A, Bigira V, et al. Comparative impacts over 5 years of artemisinin-based combination therapies on Plasmodium falciparum polymorphisms that modulate drug sensitivity in Ugandan children. J Infect Dis. 2014;210(3):344-53.

49.Bigira V, Kapisi J, Clark TD, Kinara S, Mwangwa F, Muhindo MK, et al. Protective efficacy and safety of three antimalarial regimens for the prevention of malaria in young Ugandan children: a randomized controlled trial. PLoS Med. 2014;11(8):e1001689.

50.Nankabirwa JI, Wandera B, Amuge P, Kiwanuka N, Dorsey G, Rosenthal PJ, et al. Impact of intermittent preventive treatment with dihydroartemisinin-piperaquine on malaria in Ugandan schoolchildren: a randomized, placebo-controlled trial. Clin Infect Dis. 2014;58(10):1404-12.

51.Kakuru A, Jagannathan P, Muhindo MK, Natureeba P, Awori P, Nakalembe M, et al. DihydroartemisininPiperaquine for the Prevention of Malaria in Pregnancy. N Engl J Med. 2016;374(10):928-39.

52.Nankabirwa JI, Conrad MD, Legac J, Tukwasibwe S, Tumwebaze P, Wandera B, et al. Intermittent Preventive Treatment with Dihydroartemisinin-Piperaquine in Ugandan Schoolchildren Selects for Plasmodium falciparum Transporter Polymorphisms That Modify Drug Sensitivity. Antimicrob Agents Chemother. 2016;60(10):5649-54.

53.Taylor AR, Flegg JA, Holmes CC, Guérin PJ, Sibley CH, Conrad MD, et al. Artemether-Lumefantrine and Dihydroartemisinin-Piperaquine Exert Inverse Selective Pressure on. Open Forum Infect Dis. 2017;4(1):ofw229.

54.Conrad MD, Mota D, Foster M, Tukwasibwe S, Legac J, Tumwebaze P, et al. Impact of Intermittent Preventive Treatment During Pregnancy on Plasmodium falciparum Drug Resistance-Mediating Polymorphisms in Uganda. J Infect Dis. 2017;216(8):1008-17.

55.Yeka A, Wallender E, Mulebeke R, Kibuuka A, Kigozi R, Bosco A, et al. Comparative Efficacy of Artemether-Lumefantrine and Dihydroartemisinin-Piperaquine for the Treatment of Uncomplicated 
Malaria in Ugandan Children. J Infect Dis. 2019;219(7):1112-20.

56.Sisowath C, Petersen I, Veiga MI, Martensson A, Premji Z, Bjorkman A, et al. In vivo selection of Plasmodium falciparum parasites carrying the chloroquine-susceptible pfcrt K76 allele after treatment with artemether-lumefantrine in Africa. J Infect Dis. 2009;199(5):750-7.

57.Venkatesan M, Gadalla NB, Stepniewska K, Dahal P, Nsanzabana C, Moriera C, et al. Polymorphisms in Plasmodium falciparum chloroquine resistance transporter and multidrug resistance 1 genes: parasite risk factors that affect treatment outcomes for $P$. falciparum malaria after artemether-lumefantrine and artesunate-amodiaquine. Am J Trop Med Hyg. 2014;91(4):833-43.

58.Baraka V, Tinto H, Valea I, Fitzhenry R, Delgado-Ratto C, Mbonye MK, et al. In vivo selection of Plasmodium falciparum Pfcrt and Pfmdr1 variants by artemether-lumefantrine and dihydroartemisininpiperaquine in Burkina Faso. Antimicrob Agents Chemother. 2015;59(1):734-7.

59.Veiga MI, Dhingra SK, Henrich PP, Straimer J, Gnadig N, Uhlemann AC, et al. Globally prevalent PfMDR1 mutations modulate Plasmodium falciparum susceptibility to artemisinin-based combination therapies. Nature communications. 2016;7:11553.

60.Divala TH, Mungwira RG, Mawindo PM, Nyirenda OM, Kanjala M, Ndaferankhande M, et al. Chloroquine as weekly chemoprophylaxis or intermittent treatment to prevent malaria in pregnancy in Malawi: a randomised controlled trial. The Lancet Infectious Diseases. 2018;18(10):1097-107.

\section{Tables}

Table 1. Characteristics of participants evaluated for chloroquine and lumefantrine ex vivo susceptibility assay 


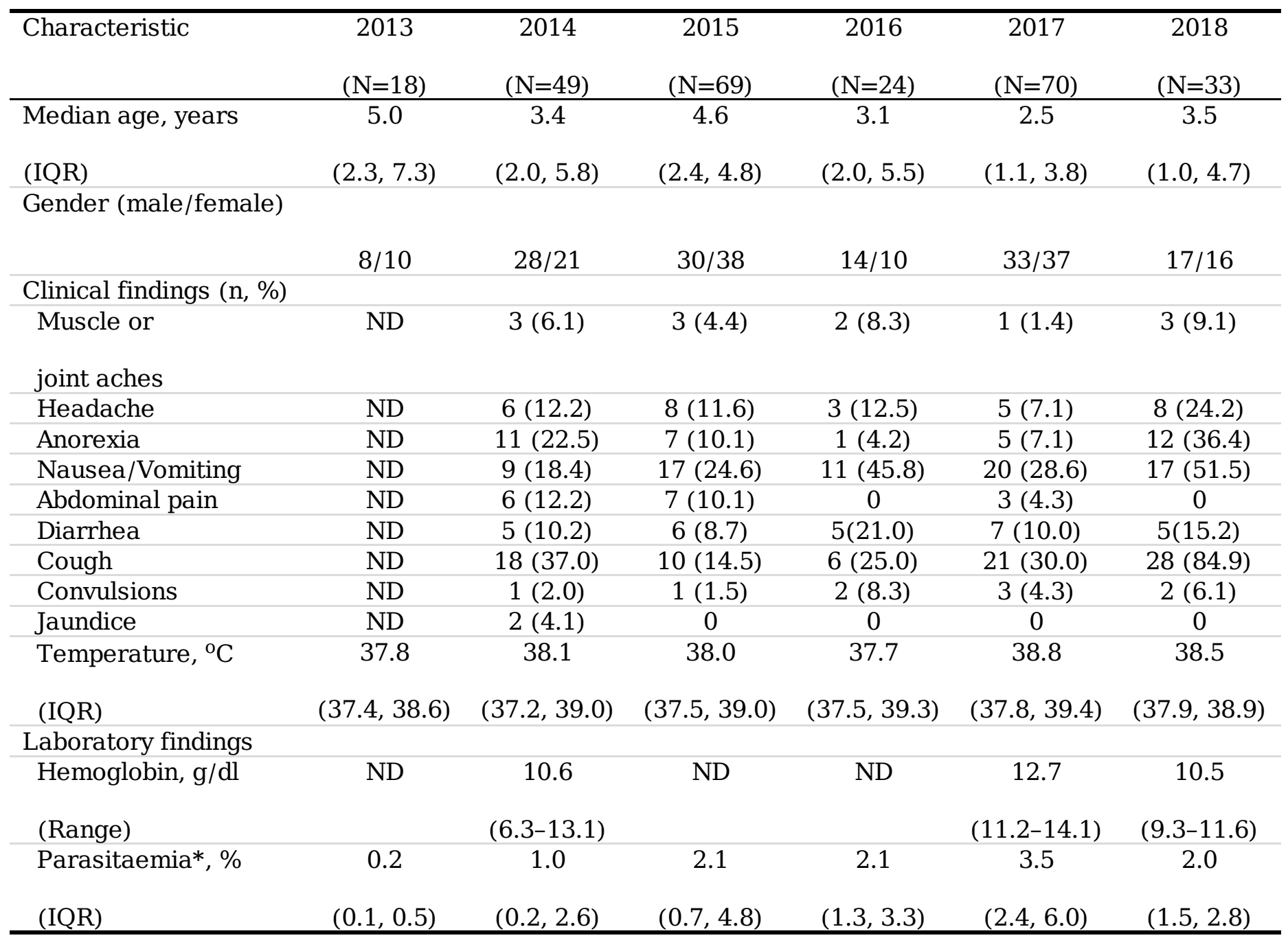

$\mathrm{ND}=$ Not Done, IQR=Inter Quartile Range, * Significant differences were observed between studied year $\left(\mathrm{p}\right.$-value $=3.9 \times 10^{-15}$, Kruskal-Wallis test).

Table 2. Chloroquine susceptibility in pfcrt haplotypes obtained from sequencing the entire pfcrt gene pfcrt polymorphic positions

\begin{tabular}{|c|c|c|c|c|c|c|c|c|c|c|c|}
\hline \multirow{2}{*}{ pfcrt Haplotypes } & \multicolumn{11}{|r|}{$\overline{\text { Mean }}$} \\
\hline & 24 & 34 & 72 & 73 & 74 & 75 & 76 & 220 & 271 & 371 & $\mathrm{CQ} \mathrm{IC}_{50}$ \\
\hline HP $1(n=31)$ & $\mathrm{D}$ & $\mathrm{R}$ & $\mathrm{C}$ & $\mathrm{V}$ & $\mathrm{M}$ & $\mathrm{N}$ & $\mathrm{K}$ & $\mathrm{A}$ & $\mathrm{Q}$ & $\mathrm{R}$ & 18.2 \\
\hline HP $2(n=4)$ & $\mathrm{Y}$ & $\mathrm{R}$ & $\mathrm{C}$ & V & M & $\mathrm{N}$ & $\mathrm{K}$ & A & $\mathrm{Q}$ & $\mathrm{R}$ & 22.2 \\
\hline HP $3(n=1)$ & $\mathrm{D}$ & $\mathrm{G}$ & $\mathrm{C}$ & V & M & $\mathrm{N}$ & $\mathrm{K}$ & A & Q & $\mathrm{R}$ & 19.7 \\
\hline HP $4(n=5)$ & $\mathrm{D}$ & $\mathrm{R}$ & $\mathrm{C}$ & V & I & $\mathrm{E}$ & $\mathrm{T}$ & S & $\mathrm{E}$ & I & 22.2 \\
\hline HP $5(n=2)$ & $\mathrm{D}$ & $\mathrm{R}$ & $\mathrm{C}$ & V & M & $\mathrm{N}$ & $\mathrm{K}$ & A & $\mathrm{E}$ & $\mathrm{R}$ & 35.2 \\
\hline HP $6(n=1)$ & $\mathrm{D}$ & $\mathrm{R}$ & $\mathrm{C}$ & $\mathrm{V}$ & M & $\mathrm{N}$ & $\mathrm{K}$ & A & Q & I & 17.5 \\
\hline
\end{tabular}


Table 3. Commonly used antimalarial drugs of patients attending St. Mary's Hospital Lacor, Gulu (information obtained from excluded patients).

\begin{tabular}{lllllll}
\hline Pretreatment Drug, $\mathrm{n}(\%)$ & 2013 & 2014 & 2015 & 2016 & 2017 & Total \\
& $(\mathrm{N}=$ & $(\mathrm{N}=$ & $(\mathrm{N}=$ & $\begin{array}{l}(\mathrm{N}= \\
(\mathrm{N}=\end{array}$ & $(\mathrm{N}=181)$ \\
& $47)$ & $96)$ & $23)$ & $8)$ & $7)$ & \\
\hline Artemether (Intramuscular) & $1(2.1)$ & 0 & 0 & 0 & 0 & $1(0.6)$ \\
Artesunate (Intravenous) & 0 & $2(2.1)$ & $2(8.7)$ & 0 & 0 & $4(2.2)$ \\
\hline Chloroquine & $3(6.4)$ & $1(1.0)$ & 0 & 0 & 0 & $4(2.2)$ \\
\hline Artemether lumefantrine & $34(72.3)$ & $67(69.8)$ & $6(26.1)$ & $7(85.7)$ & $6(85.7)$ & $120(66.3)$ \\
Sulphadoxine pyrimethamine & $2(4.3)$ & $5(5.2)$ & $1(4.4)$ & 0 & 0 & $8(4.4)$ \\
\hline Quinine & $6(12.8)$ & $6(6.3)$ & $1(4.4)$ & $1(14.3)$ & 0 & $14(7.7)$ \\
Artesunate and artemether lumefantrine & 0 & $1(1.0)$ & 0 & 0 & 0 & $1(0.6)$ \\
\hline $\begin{array}{l}\text { Artemether lumefantrine and sulphadoxine } \\
\text { pyrimethamine }\end{array}$ & 0 & $1(1.0)$ & 0 & 0 & 0 & $1(0.6)$ \\
Artemether lumefantrine and Quinine & 0 & $3(3.1)$ & 0 & 0 & 0 & $3(1.7)$ \\
\hline Unknown & $1(2.1)$ & $10(10.4)$ & $13(57.0)$ & 0 & $1(14.3)$ & $25(13.8)$ \\
\hline
\end{tabular}

\section{Figures}

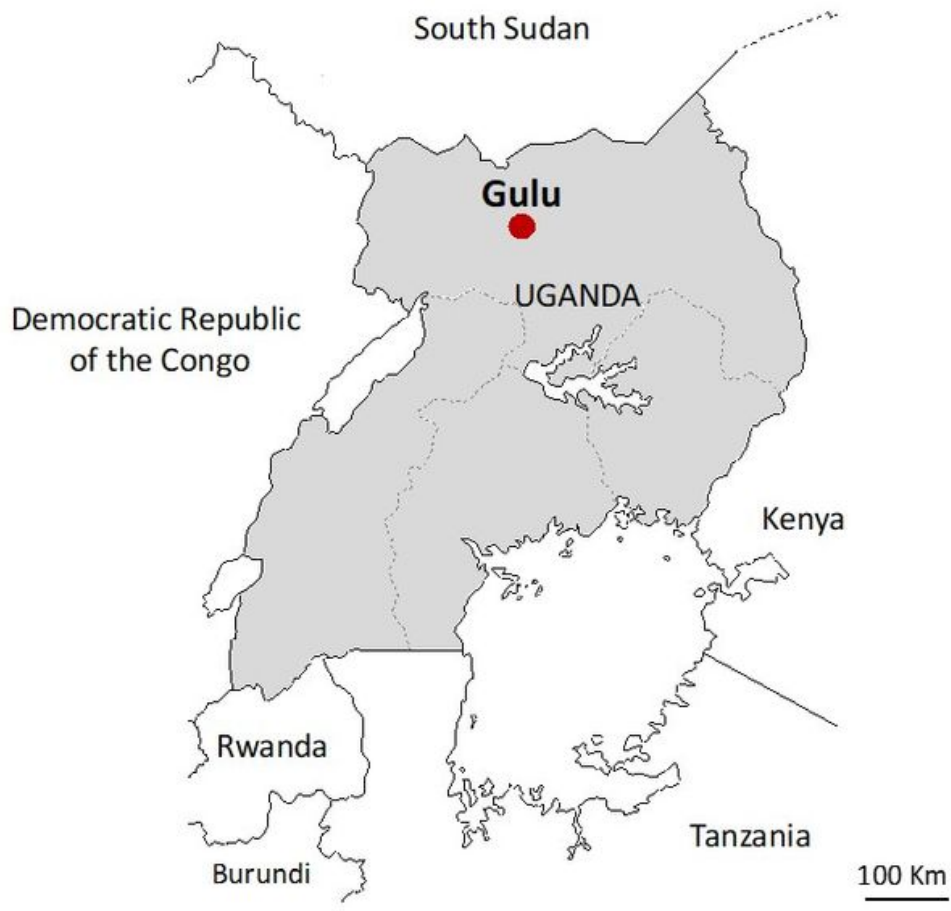

Fig 1

\section{Figure 1}




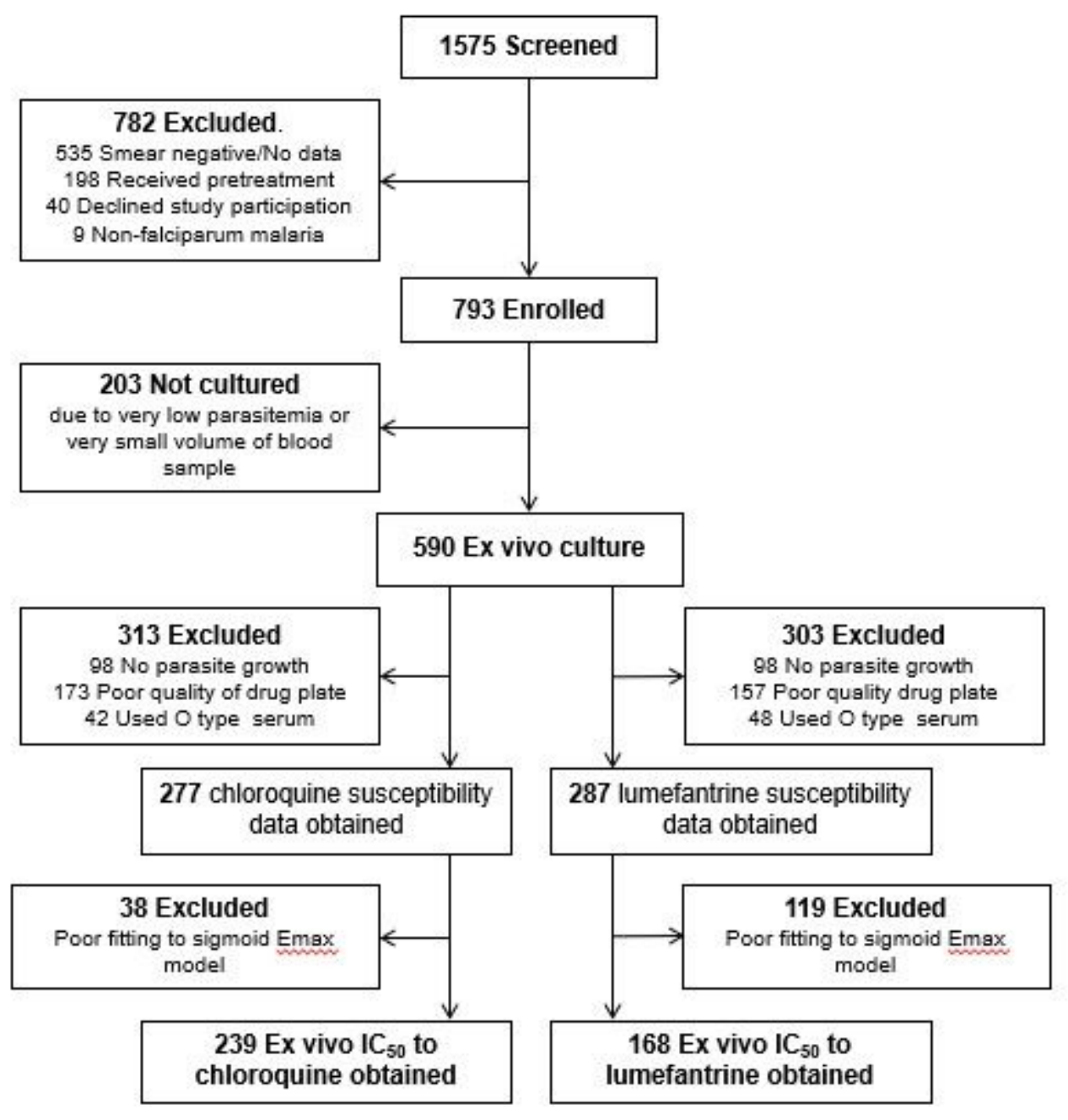

Fig 2

Figure 2

Flow chart of the study from screening to drug sensitivity assays, 2013-2018. 


\section{Fig 3}

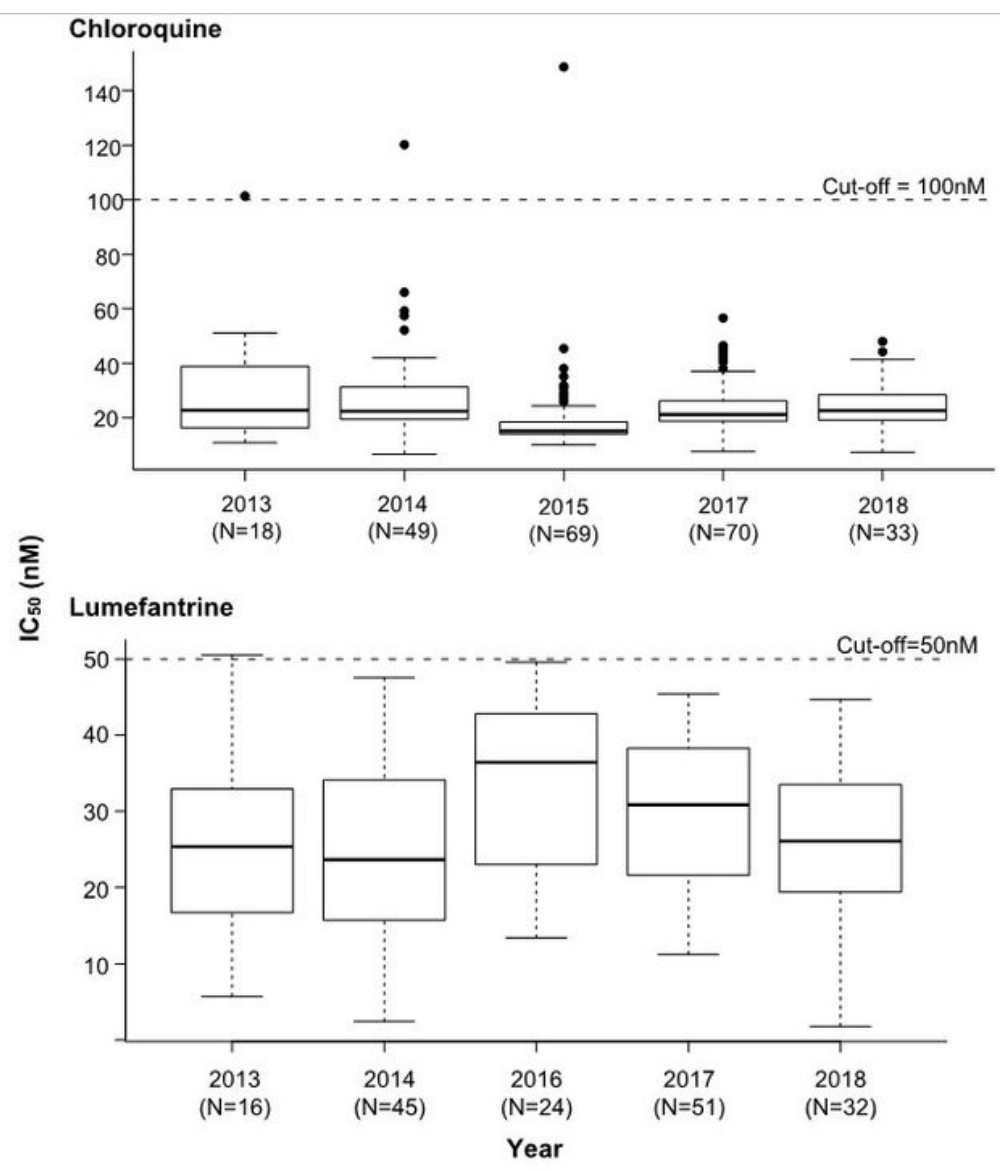

\section{Figure 3}

Ex vivo sensitivity of P. falciparum to chloroquine and lumefantrine. Bold lines represent median IC50s. Faint horizontal lines represent the 25th and 75th interquartile range. Mean IC50 for chloroquine were 24.8 nM, 24.9 nM, 17.4 nM, 22.6 nM and 23.1nM in 2013, 2014, 2015, 2017 and 2018, respectively; and for lumefantrine, 20.8 nM, 20.5 nM, 32.0 nM, 28.6 nM and 21.0 nM in 2013, 2014, 2016, 2017 and 2018 , respectively. Cut-off sensitivity is based from literature. 


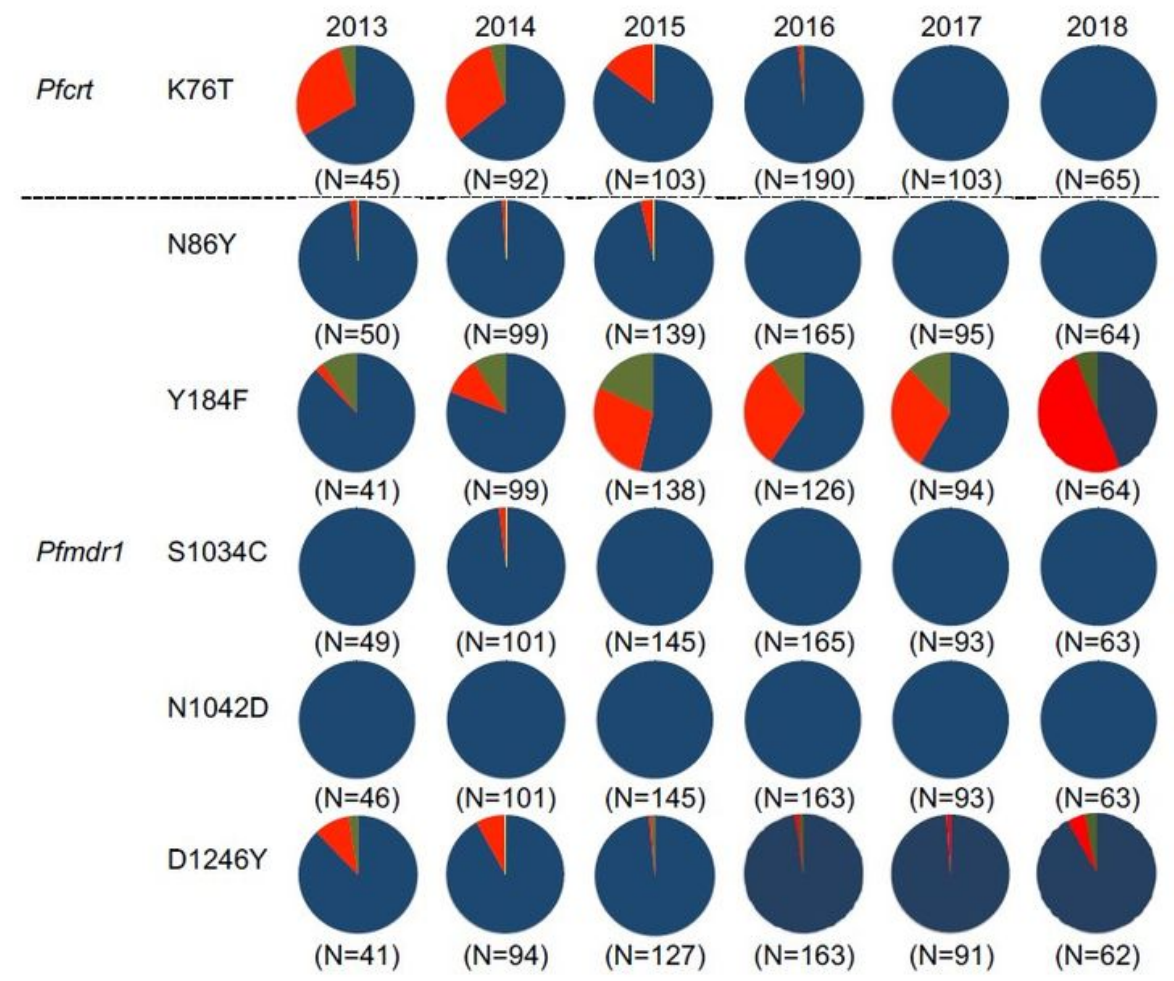

Fig 4

$\square$ Wild-type $\square$ Mixed $\square$ Mutant

Figure 4

Temporal changes in pfcrt and pfmdr1 allele prevalence in all collected P. falciparum isolates. 

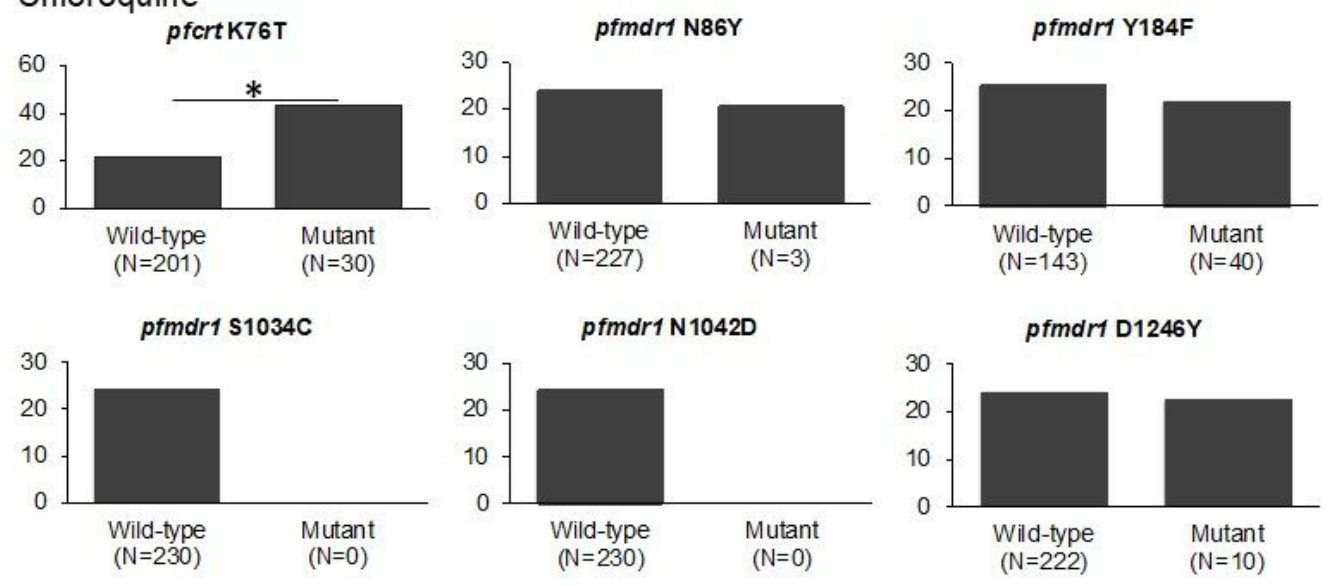

Lumefantrine
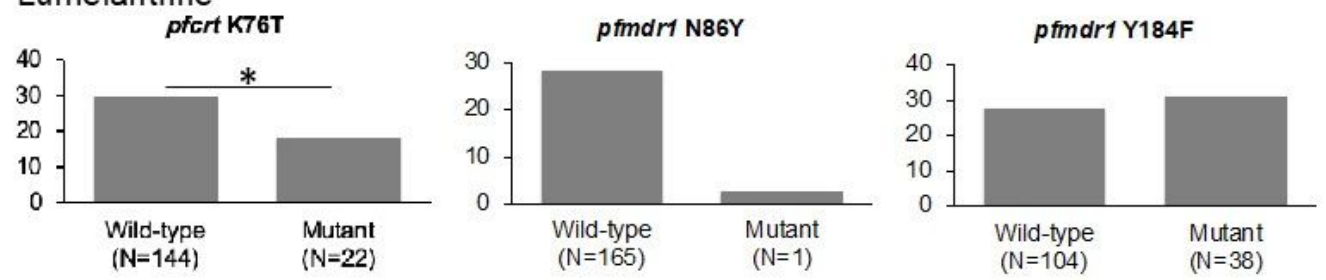

Fig 5
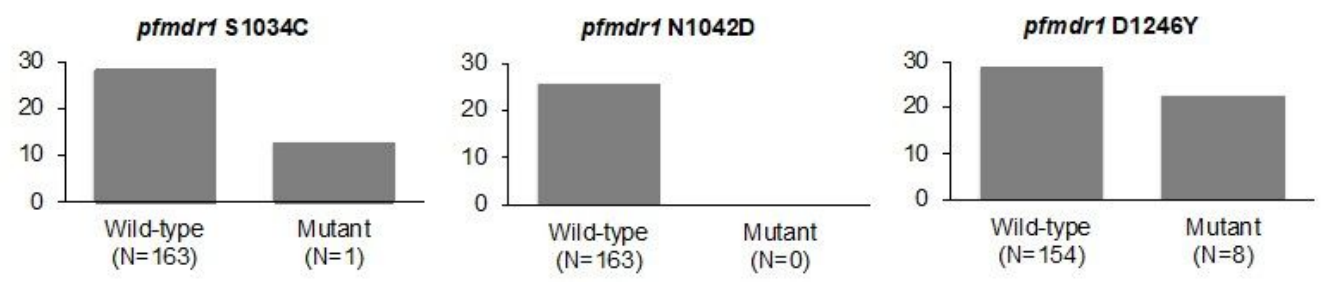

\section{Figure 5}

Association between allele prevalence and mean IC50 to chloroquine and lumefantrine. $\mathrm{N}$ is the number of samples available for comparison. Allele prevalence was compared between wild-type and mutant alleles using Wilcoxon rank sum test, and comparisons with $P$ values of $<0.05$ are significant. $P$ values $<0.0001$ are indicated by *. 